\title{
Uncertainty quantification analysis of RANS of spray jets
}

\author{
Pietro Paolo Ciottoli*1 ${ }^{*}$, Andrea Petrocchi ${ }^{\dagger 1}$, Riccardo Malpica Galassi ${ }^{\ddagger 1}$, Lorenzo Angelilli ${ }^{\S 1,2}$, Francisco E. \\ Hernández Pérez ${ }^{\mathbb{I} 2}$, Francesco Picano ${ }^{\| 3}$, Mauro Valorani**1, and Hong G. Im ${ }^{\dagger \dagger 2}$ \\ ${ }^{1}$ Sapienza, Università di Roma, Italy \\ ${ }^{2}$ King Abdullah University of Science and Technology (KAUST), CCRC, Thuwal 23955-6900, Saudi Arabia \\ ${ }^{3}$ Department of Industrial Engineering \& CISAS, University of Padova, Italy
}

\begin{abstract}
Parametric uncertainty is propagated through Reynolds-averaged Navier-Stokes (RANS) computations of a prototypical acetone/air aerosol stream flowing in a dry air environment. Two parameters are considered as uncertain: the inflow velocity dissipation and a coefficient that blends the discrete random walk and the gradient-based dispersion models. A Bayesian setting is employed to represent the degree of belief about the parameters of interest in terms of probability theory, such that uncertainty is described with probability density functions. Random variables are represented by means of polynomial chaos expansions. The sensitivity of mean axial velocity and mean vapor mass fraction to the uncertain parameters is discussed.
\end{abstract}

\section{Introduction}

This work is part of a wider effort to construct a numerical framework devoted to the prediction of combustion and heat transfer phenomena in throttleable, pintle-injector-based, oxygen-methane liquid rocket engine (LRE) thrust chambers. Hence, we aim at extending the present computational fluid dynamics (CFD) capabilities to deal with multiphase reacting flows and complex thermodynamics, with the final goal of developing an innovative and validated numerical toolkit of analysis that is able to abate the development cost of the next generation LRE combustion chambers by allowing "virtual experiments" in place of actual experimental testing in the lab [1, 2]. When operated at subcritical pressure, the fuel and/or oxidizer injection in the combustion chamber occurs in a liquid state, and primary and secondary liquid breakup as well as subsequent droplet evaporation and dispersion in a turbulent environment need to be accounted for in numerical simulations. As such, the pressure and temperature conditions can be far from ideal, and proper real-gas thermodynamic models are required [3, 4]. However, the excessive computational effort required to capture the small-scale dynamics typical of turbulent reacting flows forces us to resort to lower-fidelity approaches such as large eddy simulations (LES) [5, 6], detached eddy simulations (DES) [7, 8] and Reynolds-averaged Navier-Stokes (RANS) computations, here ordered by decreasing fidelity level and required computational effort. Furthermore, the use of CFD for design and optimization, which requires multiple model evaluations, brings the need for robust and computationally affordable numerical methods, often leaving RANS as the only viable option with the present computational capabilities [9].

In the RANS context, only the ensemble averages of the gas-phase kinematic and thermodynamic state are resolved, while the deviations around the mean values are modeled. The liquid-phase is often treated in a Lagrangian fashion, which consists in evolving parcels, i.e., ensembles of droplets sharing common mean properties, in space and time. The numerical models describing the mean properties of both the Eulerian gas-phase and the Lagrangian liquid-phase are characterized by a wide number of tunable parameters, often requiring proper calibration. Unfortunately, the severe thermodynamic conditions typical of this class of flows often result in a lack of data availability, which prevents proper calibration and leads to uncertainty in the parameter values. The presence of parametric uncertainty, if not taken into account, lowers the reliability of predictions, undermining their usefulness in the design process. A way to circumvent this issue is to adopt uncertainty quantification (UQ) techniques to estimate the uncertainty in numerical predictions induced by the presence of calibrated parameters in underlying models [10]. UQ techniques are rather expensive, since

\footnotetext{
*Researcher fellow, Department of mechanical and aerospace engineering, pietropaolo.ciottoli@uniroma1.it

${ }^{\dagger}$ Research assistant, Department of mechanical and aerospace engineering, petrocchi.1661636@studenti.uniroma1.it, AIAA student member

†Postdoctoral fellow, Department of mechanical and aerospace engineering, riccardo.malpicagalassi@uniroma1.it

$\S$ PhD Student, corresponding author, Clean Combustion Research Center, lorenzo.angelilli@kaust.edu.sa, AIAA Student member

IIResearch scientist, Clean Combustion Research Center, francisco.hernandezperez.1@kaust.edu.sa, AIAA Member

॥Associate professor, Department of Industrial Engineering, francesco.picano@unipd.it

** Professor, Department of mechanical and aerospace engineering, mauro.valorani@ uniroma1.it

$\dagger$ Professor, Clean Combustion Research Center, hong.im@kaust.edu.sa, AIAA Associate fellow
} 
a single model evaluation is not sufficient to estimate predictive uncertainties, unless the forward model is recast to solve directly for probabilistic quantities, hence multiple simulations with black-box numerical codes are required. The focus is thus shifted from favoring high fidelity in a single simulation, which does not carry any reliability estimate, to seeking confidence through uncertainty bounds with lower-fidelity, affordable predictions [11]. Similar to experimental measurements, the numerical results are hence accompanied by a confidence interval. Moreover, sensitivity information is naturally available within the UQ framework, which allows to quantify the impact of each uncertain parameter on the variability of the predicted quantities of interest [12].

The goal of this paper is to propagate the uncertainty associated with selected RANS models and to assess (i) the variability in typical flow observables and (ii) the sensitivity of the flow observables to each uncertain parameter. We perform this uncertainty propagation in a prototypical flow configuration, which is a saturated acetone/air aerosol stream flowing in a dry air environment. The test case was already characterized via direct numerical simulation (DNS) [13, 14] and is here replicated through a RANS approach coupled with a realizable k-epsilon model. Two parameters are considered as uncertain: the inflow turbulent kinetic energy dissipation rate $\epsilon_{0}$ and a coefficient $\alpha$ that blends two well-established dispersion models, namely the discrete random walk and the gradient-based dispersion models. We employ a Bayesian setting to represent the degree of belief about the parameters of interest in terms of probability theory, such that uncertainty is described with probability density functions (PDF). Polynomial chaos expansions (PCE) are employed to represent random variables (RVs). PCEs allow efficient propagation of uncertainty in computational models.

\section{A. Numerical setup and test case description}

The RANS Eulerian-Lagrangian numerical approach [15,-20] is described in this section. The ensemble averages of the turbulent Eulerian carrier phase state vector is governed by the following system of equations:

$$
\begin{gathered}
\frac{\partial \bar{\rho} \tilde{y}_{\beta}}{\partial t}+\frac{\partial}{\partial x_{j}}\left[\bar{\rho} \tilde{y}_{\beta} \tilde{u_{j}}+J_{j}+\bar{\rho} \overline{u_{j}^{\prime} y_{\beta}^{\prime}}\right]=\bar{S}_{m}^{\beta^{D}} \quad \text { for } \quad \beta=1, \ldots, N, \\
\frac{\partial\left(\bar{\rho} \tilde{u}_{i}\right)}{\partial t}+\frac{\partial}{\partial x_{j}}\left[\bar{\rho} \tilde{u}_{i} \tilde{u}_{j}+\bar{p} \delta_{i j}-\tilde{\tau}_{i j}+\overline{\rho u_{i}^{\prime} u_{j}^{\prime}}\right]=\bar{S}_{p, i}^{D}, \\
\frac{\partial \bar{\rho} \tilde{e}}{\partial t}+\frac{\partial}{\partial x_{j}}\left[\bar{\rho} \tilde{e} \tilde{u}_{j}+\tilde{u}_{j} \bar{p}+\tilde{q}_{j}-\tilde{u}_{i} \tilde{\tau}_{i j}\right]=\bar{S}_{e}^{D}
\end{gathered}
$$

where the "overbar" represents the Reynolds average, while the "tilde" represents Favre-average $(\widetilde{\phi}=\overline{\rho \phi} / \bar{\rho}), \rho$ is the density, $y$ is the mass fraction of the species $\beta, J_{j}$ is the diffusive term, $u_{i}$ is the velocity in direction $i, p$ is the pressure, $\tau$ is the viscous stress tensor, $e$ is the internal energy and $q$ is the heat transfer rate. The term $\overline{\rho u_{i}^{\prime} u_{j}^{\prime}}$ represents the Reynolds stress tensor, modeled via the realizable $k-\epsilon$ (RKE) two-equation model [21]. The present model yields better predictions than the standard $k-\epsilon$ model, overcoming the well-known spreading rate anomaly for jets. In RKE a modified equation for turbulent dissipation is employed [22] to ensure a proper description of jet dynamics, while the turbulent kinetic energy equation is the same as that of the standard version. The right-hand-side (RHS) terms are the coupling terms, representing the contribution of Lagrangian droplets with regard to species mass, momentum, and energy on the Eulerian gaseous medium. These are expressed as:

$$
\begin{gathered}
S_{m}^{\beta^{D}}=-\sum_{k=1}^{n_{d}} \dot{m}_{d}^{\beta^{k}}(t) \delta\left(x-X_{d}^{k}(t)\right), \\
S_{p}^{D}=-\sum_{k=1}^{n_{d}} U_{d}^{k}(t) \dot{m}_{d}^{\beta^{k}}(t) \delta\left(x-X_{d}^{k}(t)\right) \\
S_{e}^{D}=-\sum_{k=1}^{n_{d}} \sum_{\beta=1}^{N}\left(h_{d}^{\beta^{k}}(t) \dot{m}_{d}^{\beta^{k}}(t)+\dot{h}_{d}^{\beta^{k}}(t) m_{d}^{\beta^{k}}(t)\right) \delta\left(x-X_{d}^{k}(t)\right)
\end{gathered}
$$


The Lagrangian evolution equations governing the motion of the dispersed phase are reported in the following:

$$
\begin{aligned}
\frac{d m_{d}^{\beta^{k}}}{d t} & =\dot{m}_{d}^{\beta^{k}}, \\
\frac{d \mathbf{U}_{d}^{k}}{d t} & =\frac{\mathbf{F}}{m_{d}^{k}}, \\
\frac{d h_{d}^{\beta^{k}}}{d t} & =\dot{h}_{d}^{\beta^{k}}, \\
\frac{d \mathbf{X}_{d}^{k}}{d t} & =\mathbf{U}_{d}^{k},
\end{aligned}
$$

describing the conservation of mass (eq. 77), momentum (eq. 8), and energy (eq. 9p, as well as the droplets trajectory (eq. 10). The RHS of eqs. (7)-(9) represent the forcing terms and accounts for droplet evaporation, aerodynamic forces, and convective heat exchange. The aerodynamic force in eq. (8), consisting of drag only, is modeled as $\mathbf{F}=\left(C_{D} / \tau_{d}\right)\left(\mathbf{u}_{\text {gas }}-\mathbf{U}_{d}^{k}\right)\left|\mathbf{u}_{\text {gas }}-\mathbf{U}_{d}^{k}\right|$ where the drag coefficient $C_{D}$ is function of the finite droplet Reynolds number $\operatorname{Re}_{d}$ by means of the Schiller-Neumann correlation [15]. The mass vaporization rate in eq. (7) is written through the Sherwood number Sh, while the convective heat exchanged in eq. (9) is related to the Nusselt number Nu: $\dot{m}_{d}^{\beta}=2 \pi r_{d} \operatorname{Sh} D \rho \ln (1+B)$ and $h_{d}^{\beta}=\mathrm{Nu}_{d} \alpha / L$. The latter can be expressed as a function of the droplet Reynolds number through the Frossling correlations [15], which account for the mass diffusivity and the thermal conductivity through the Schmidt and Prandtl numbers, respectively: $\mathrm{Sc}=\mu /(\rho D)$ and $\operatorname{Pr}=\mu /\left(c_{p} k\right)$.

\section{B. Dispersion model}

The Lagrangian momentum equation (eq. 8) has aerodynamic drag as a forcing term, this being function of the carrier phase velocity ( $\mathbf{u}_{\text {gas }}$ ) relative to the Lagrangian droplet position. Within the RANS approach, the turbulent fluctuations are not directly resolved, hence droplets subject to the resolved velocity would be influenced by the mean velocity field only. Therefore, a droplet would not undergo the expected physical spatial turbulent oscillation, leading to a lack of accuracy in terms of both location and intensity of the evaporation. The introduction of a dispersion model is necessary for a satisfactory reconstruction of the velocity field of the carrier phase in the droplet position. The gas phase velocity can be written as the summation of two contributions $\left(\mathbf{u}_{\text {gas }}=\overline{\mathbf{u}}+\mathbf{u}^{\prime}\right)$ : (i) the resolved field directly computed by the Eulerian system ( $\overline{\mathbf{u}})$ and (ii) an additional component related to the unresolved fluctuations $\left(\mathbf{u}^{\prime}\right)$. The role of the dispersion model is to provide an estimate of the $\mathbf{u}^{\prime}$ component. Two different dispersion models for RANS are explored in this work: (i) discrete random walk (DRW) [23] and (ii) gradient-based dispersion. In the DRW model the fluctuation velocity vector components are expressed as follows:

$$
u_{i, D R W}^{\prime}=\lambda_{i} \sqrt{\frac{2}{3} k}
$$

where $k$ is the turbulent kinetic energy and $\lambda_{i}$ are random Gaussian variables, having zero mean and unity standard deviation. The random distribution of the velocity vector directions is justified by the hypothesis of isotropic turbulence. In the gradient-based dispersion model it is assumed that the vector $\mathbf{u}^{\prime}$ has the same direction of the turbulent kinetic energy gradient $\nabla k$ :

$$
\mathbf{u}_{\nabla}^{\prime}=\gamma \frac{\nabla k}{|\nabla k|} \sqrt{\frac{2}{3} k}
$$

where $\gamma$ is randomly imposed to plus or minus one.

A stochastic model for the velocity oscillation is defined following the work of Mueller and Raman [24], who employed the peer model in the LES context to inquire the sensitivity of a reactive jet flame to the modeled sub-filter mixture fraction dissipation. The velocity oscillation is then defined as:

$$
\mathbf{u}^{\prime}=(1-\alpha) \mathbf{u}_{D R W}^{\prime}+\alpha \mathbf{u}_{\nabla}^{\prime}
$$

where $\alpha$ is a stochastic parameter that sets the contribution of each of the two dispersion models. 


\section{Test case description}

The computations are based on the test case inquired via DNS by Dalla Barba et al. [13, 14]. The test case consists of a cylindrical domain filled with air in non-reactive conditions where a multi-phase acetone-air jet flows out of an orifice at the center of one base and acetone droplets are randomly dispersed through the inflow section. The gas-vapor mixture is injected into an open environment through an orifice of radius $\mathrm{R}=5 \cdot 10^{-3} \mathrm{~m}$ at a bulk axial velocity $U_{x, 0}=8.1 \mathrm{~m} / \mathrm{s}$. The distribution of droplets on the inflow section consists of a random position and velocity distribution of monodisperse, liquid-acetone droplets with an initial radius $r_{d, 0}=6 \mu \mathrm{m}$. The ambient pressure is set to $p_{0}=101325 \mathrm{~Pa}$, the injection temperature is fixed to $T_{0}=273.15 \mathrm{~K}$ for both the carrier and the dispersed phases. The injection flow rate of the gaseous phase is kept constant fixing a bulk Reynolds number $R e=2 U_{x, 0} R / v=6000$, $v=1.35 \cdot 10^{-5} \mathrm{~m}^{2} / \mathrm{s}$ being the kinematic viscosity. A nearly-saturated condition is prescribed for the air-acetone vapor mixture at the inflow section, $S=Y_{v} / Y_{v, s}=0.99$, where $S$, is the saturation, $Y_{v}$ is the actual vapor mass fraction on the inflow section and $Y_{v, s}\left(p_{0}, T_{0}\right)$ is the vapor mass fraction in fully-saturated condition evaluated at the inflow temperature and pressure. The acetone to air mass-flow-rate ratio is set to $\Psi=\dot{m}_{a c t} / \dot{m}_{a i r}=0.28, \dot{m}_{a c t}=\dot{m}_{a c t, l}+\dot{m}_{a c t, v}$ being the sum of the liquid, $\dot{m}_{a c t, l}$ and gaseous, $\dot{m}_{a c t, l}$, acetone mass-flow-rates. This configuration corresponds to a bulk volume fraction of the liquid phase $\Phi=8 \cdot 10^{-5}$.

The RANS computations were performed by means of the OpenFOAM-based sprayFoam solver. A backward implicit Euler scheme was employed for the time integration. A Gaussian second-order integration with linear interpolation was used for the gradient terms, while two schemes were used for divergence terms: an upwind interpolation was used for $u$, $p, k, \epsilon_{0}$ and $Y$, whereas a linear approach was used for the rest of the divergence terms in the energy equation. Finally, a Gauss linear scheme was employed for Laplacian operators with orthogonal-corrected schemes for gradients.

The average profiles of the DNS of Dalla Barba et al. [13] are imposed as inflow conditions of velocity and turbulent kinetic energy $k$ while the reference value of the inflow turbulent kinetic energy dissipation rate $\epsilon_{0}$ is evaluated at each point of the inflow section as $\epsilon_{0}=C_{\mu}^{3 / 4} k^{3 / 2} / l_{m}$, where $l_{m}$ is the nominal $l_{m}=0.07 * 2 R$ and $C_{\mu}$ is the same coefficient used to evaluate the eddy viscosity in the two-equation closure model. A preliminary mesh sensitivity study, as well as a parametric campaign, varying the number of droplets per parcel, was performed. As a result, the computational domain was discretized with a mesh consisting of 136000 cells, and each Lagrangian particle represents a parcel of 100 droplets, without affecting the solution accuracy. The numerical setup allowed to perform a complete RANS simulation in less than two hours on 20 cores.

\section{Uncertainty quantification and polynomial chaos expansion}

This work aims at inquiring how the uncertainty in parameters characterizing the mathematical model propagates on the quantities of interest (QoI). In our specific case, we selected two parameters. The first is the dissipation rate value at the jet inflow $\epsilon_{0}$. The second is the factor $\alpha$ blending the gradient-based and the purely stochastic dispersion models, as defined in eq. 13 . This goal is achieved relying on the Bayesian setting, representing the degree of belief about the parameters of interest in terms of probability theory, such that uncertainty is described with probability density functions (PDF).

The inflow dissipation $\epsilon_{0}$ is presumed to have a Gaussian distribution. At each point of the inflow section, the mean value is chosen as per the well-known relation between the turbulent kinetic energy and dissipation in a turbulent pipe $\mu\left(\epsilon_{0}\right)=C_{\mu}^{3 / 4} k^{3 / 2} / l_{m}$ introduced above. The standard deviation $\sigma_{\epsilon_{0}}$ is chosen to be $10 \%$ of the mean value, meaning that the most probable value is the one resulting from the known relation, and a degree of uncertainty is retained. On the other hand, the blending coefficient $\alpha$ can vary between zero and one, letting the dispersion model vary from purely gradient-based to purely stochastic. We presume a uniform PDF for $\alpha$ in the range [0,1], since there is no reason to prefer one model over the other. The two parameters $\epsilon_{0}$ and $\alpha$ are assumed to be uncorrelated.

Since the PDFs of the input parameters are known, the most straightforward approach would be to sample values of the input parameters and obtain the corresponding quantities of interest via RANS computations in a Monte Carlo fashion. If a sufficiently large number of samples is produced, the statistics of the QoIs can be computed. Once the PDFs of the QoIs are known, it is straightforward to compute: the main effect sensitivity, measuring the variance contribution solely due to the i-th parameter $S_{i}$; the Joint effect sensitivity $S_{i j}$, measuring the variance contribution from the interaction of $\mathrm{i}-t h$ and $\mathrm{j}-t h$ parameters; and the total effect sensitivity $\mathrm{S}_{T, i}$, measuring the variance contributions from all terms that involve the $\mathrm{i}-t h$ parameter. However, the convergence rate of the Monte Carlo methods with the number of samples is notoriously slow: to accurately characterize the statistical properties of the QoIs, the procedure described above requires a large number of samples, typically of the order of thousands, each requiring a RANS computation. This issue can be circumvented by assuming a certain degree of smoothness in the QoIs statistics and resorting to a 
non-intrusive spectral projection technique employing a polynomial chaos expansions (PCE) representation of the uncertain variables. PCEs are typically employed to describe Random Variables (RVs). A PCE provides a useful means for propagating uncertainty as an alternative to classical Monte Carlo methods and allows efficient propagation of uncertainty in computational models and straightforward assessment of output variance sensitivity to the uncertain inputs $\lambda$, which in our case are $\lambda=\left\{\epsilon_{0}, \alpha\right\}$.

The uncertain input parameters $\lambda$ can be written as function of independent identically distributed (i.i.d.) standard random variables $\xi_{i}$. Hence $\lambda=\lambda(\xi)$, where $\xi=\left(\xi_{1}, . ., \xi_{n_{s}}\right)$, are random variables and $n_{s}$ usually equals the number of the stochastic degrees of freedom. In our case we have two uncertain parameters, $\epsilon_{0}$ and $\alpha$, and we chose to have two random variables $\xi_{1}$ and $\xi_{2}$ such that $\epsilon_{0}=\epsilon_{0}\left(\xi_{1}\right)$ and $\alpha=\alpha\left(\xi_{2}\right)$. Choosing $\xi_{1}=\mathcal{N}(0,1)$ to be a normal distribution of mean 0 and variance 1 , and $\xi_{2}=\mathcal{U}(-1,1)$ a uniform distribution, we can write the uncertain parameters as:

$$
\begin{aligned}
& \epsilon_{0}\left(\xi_{1}\right)=\mu_{\epsilon_{0}}+\xi_{1} \sigma_{\epsilon_{0}}, \\
& \alpha\left(\xi_{2}\right)=\mu_{\alpha}\left(1+\xi_{2}\right),
\end{aligned}
$$

where $\mu_{\epsilon_{0}}$ and $\sigma_{\epsilon_{0}}$ are the mean and standard deviation of $\epsilon_{0}$ and $\mu_{\alpha}$ is equal to 0.5. Being the QoI $\mathbf{y}$ function of the input parameters, they also depend on the random variables $\boldsymbol{\xi}$, so that: $\boldsymbol{y}=\boldsymbol{y}\left(\xi_{1}, \xi_{2}\right)$.

A PCE is a spectral representation of a random variable $\lambda(\xi)$ or $\mathbf{y}(\xi)$ as:

$$
\begin{aligned}
& \lambda \simeq \sum_{\beta \in J} \lambda_{\beta} \boldsymbol{\Psi}_{\beta}\left(\xi_{1}, \ldots, \xi_{n_{s}}\right) \\
& y \simeq \sum_{\beta \in J} y_{\beta} \boldsymbol{\Psi}_{\beta}\left(\xi_{1}, \ldots, \xi_{n_{s}}\right)
\end{aligned}
$$

where $\beta=\left(\beta_{1}, \ldots, \beta_{n_{s}}\right), \forall \beta_{j} \in \mathbb{N}_{0}$, is a multi-index; and $\boldsymbol{\Psi}_{\beta}$ are multivariate normalized orthogonal polynomials written as products of univariate orthonormal polynomials $\psi_{\beta}$ :

$$
\boldsymbol{\Psi}_{\beta}\left(\xi_{1}, \ldots, \xi_{n_{s}}\right)=\prod_{j=1}^{n_{s}} \psi_{\beta_{j}}\left(\xi_{j}\right)
$$

The univariate functions $\psi_{\beta}$ are polynomials of degree $\beta_{j}$ in the independent variable $\xi_{j}$ and are orthonormal with respect to the density of the random variable $\xi$, such that:

$$
\mathbb{E}\left[\psi_{k}(\xi) \psi_{n}(\xi)\right]=\int \psi_{k}(\xi) \psi_{n}(\xi) p(\xi) d \xi=\delta_{k, n}
$$

In our case, we chose Hermite polynomials for the $\xi_{1}$ direction and the Legendre polynomials for $\xi_{2}$. The coefficients $\lambda_{\beta}$ and $y_{\beta}$ are the expansion coefficients and are obtained by Galerkin projection. As an example, the $y_{\beta}$ are defined as :

$$
y_{\beta}=\mathbb{E}\left[y(\lambda) \psi_{\beta}\right]=\int y(\lambda(\xi)) \psi_{\beta}(\xi) p(\xi) d \xi
$$

It should be stressed that once the proper basis $\boldsymbol{\Psi}$ is chosen, the computation of the coefficients $y_{\beta}$ with eq. 20 allows to fully characterize the uncertain output $y$. In this work, we compute the coefficients resorting to a quadrature technique, that allowed to approximate the integral with the knowledge of $y\left(\lambda\left(\xi_{i}\right)\right)$, where $\xi_{i}$ are the quadrature points. In our case, we employed a $3 \times 3$ discretization of the sample space $\xi_{1}, \xi_{2}$, for a total of 9 simulations. The chosen quadrature points are those required for a Gaussian and a uniform distributions. The PCE approach brings the possibility to compute the sensitivity indices as follows:

$$
\begin{gathered}
\mathbb{E}[y(\xi)]=y_{0} \\
\operatorname{Var}[y(\xi)]=\sum_{\beta>0}^{N} y_{\beta}^{2}\left\langle\psi_{\beta}, \psi_{\beta}\right\rangle \\
S_{i}=\frac{1}{\operatorname{Var}[y(\xi)]} \sum_{\beta \in J_{i}}^{N} y_{\beta}^{2}\left\langle\psi_{\beta}, \psi_{\beta}\right\rangle, \quad \text { where } \quad J_{i}=\left\{\beta \in J: \beta_{i}>0, \beta_{k}=0, k \neq i\right\}
\end{gathered}
$$



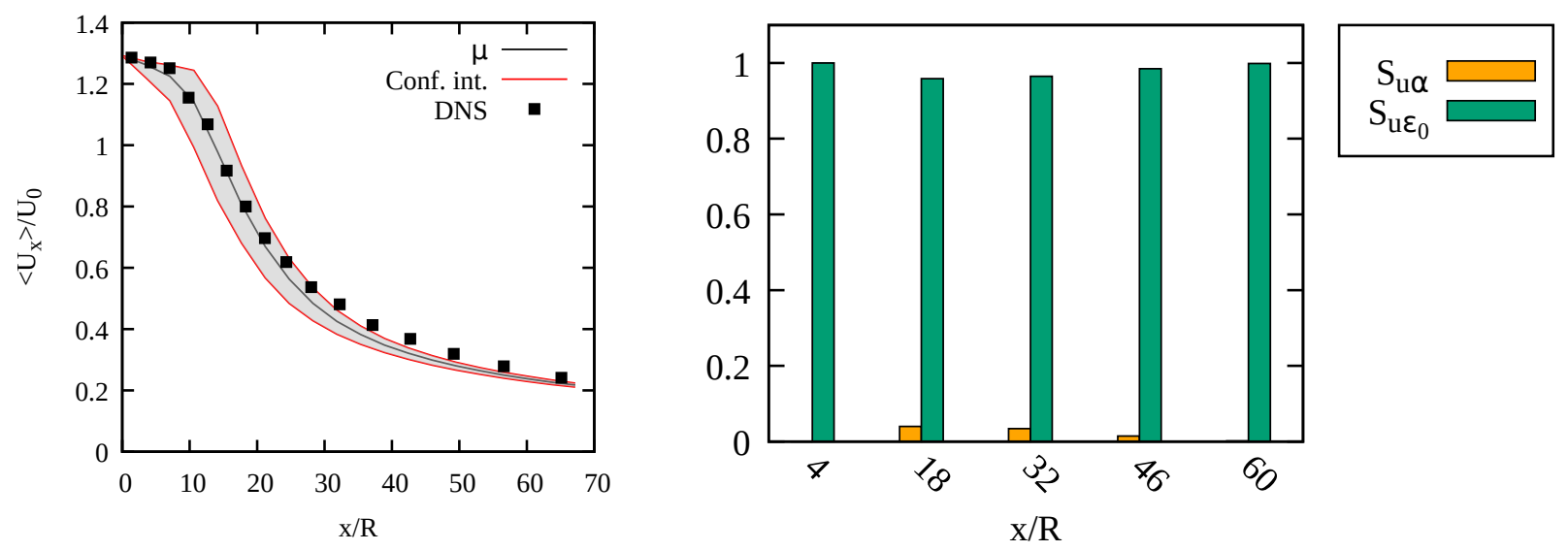

Fig. 1 Left: mean axial velocity axial distribution. Red lines encompass a grey region, representing the confidence interval. The reference DNS data are reported as black dots. The axial velocity is scaled with the bulk inflow velocity $U_{0}$. Right: influence coefficients $S_{u \alpha}$ and $S_{u \epsilon_{0}}$ as a function of the nondimensional axial distance $x / R$.

$$
S_{i j}=\frac{1}{\operatorname{Var}[y(\xi)]} \sum_{\beta \in J_{i j}}^{N} y_{\beta}^{2}\left\langle\psi_{\beta}, \psi_{\beta}\right\rangle, \quad \text { where } \quad J_{i}=\left\{\beta \in J: \beta_{i}>0, \beta_{j}>0, \beta_{k}=0, k \neq i, k \neq j\right\} .
$$

We chose a Gaussian distribution for $\epsilon_{0}$ with mean value $\mu_{\epsilon_{0}}=23.66 \mathrm{~m}^{2} / \mathrm{s}^{3}$ and standard deviation $\sigma_{\epsilon_{0}}=2.366 \mathrm{~m}^{2} / \mathrm{s}^{3}$, and a uniform distribution for $\alpha$ with mean $\mu_{\alpha}=0.5$. The sensitivity coefficients reported in the following section were computed by means of eq. 233 and eq. 24p. Nonetheless only $S_{i}$ are reported, being the $S_{i j}$ coefficients negligible everywhere in the field.

\section{Results and discussion}

The uncertain RANS solutions pdfs are computed via the PCE approach as detailed in sec. I.D These are then employed to calculate the mean values and the variance of the QoIs, respectively, and the QoIs confidence interval, defined as the interval of values containing the $90 \%$ of the QoI probability. The reference DNS data [13, 14] are also used as a reference. We compare and discuss two quantities of interest, namely the mean axial velocity scaled by the bulk inflow velocity $\left\langle U_{x}\right\rangle / U_{0}$, and the mean vapor concentration scaled by the saturated vapor concentration $\left\langle Y_{v}\right\rangle / Y_{v, s}$. The spatial distances are scaled by the jet radius $\mathrm{R}$.

The axial distributions of nondimensional mean axial velocity $\left\langle U_{x}\right\rangle / U_{0}$ are shown in Fig. 11(left). In the figure, red lines encompass a grey region, representing the confidence interval. The reference DNS data are reported as black dots. Firstly, the agreement between the mean values of the axial velocity and the reference DNS data is satisfactory, although not perfect, also recalling that the points outside the confidence interval are less than $10 \%$ likely to occur varying the two uncertain parameters of choice. Hence, if reference DNS data lie outside the confidence interval, this behavior cannot be captured by the RANS with an improved calibration of the uncertain parameters under investigation, and the cause of the mismatch should be found elsewhere in the numerical model. In particular, in Fig. 11(left), we observe a good agreement of the mean axial velocity $\left\langle U_{x}\right\rangle / U_{0}$ profile and the DNS data in the region where $x / R<20$ and a minor under-prediction further downstream. We also observe that the confidence interval, strictly related to the standard deviation of the solution $\sigma\left(\left\langle U_{x}\right\rangle / U_{0}\right)$, is negligible at the inflow, pointing out that the inflow Dirichlet condition is insensitive to the uncertain parameters. The confidence interval then increases symmetrically around the mean values for the first ten radii from the inflow and decreases further away. Observing the influence coefficients $S_{u \alpha}$ and $S_{u \epsilon_{0}}$, reported in Fig. 1 (right), we deduce that the axial velocity is mostly influenced by the inflow value of $\epsilon_{0}$, being $S_{u \alpha}$ null or negligible everywhere along the jet axis. Moreover, a change in $\epsilon_{0}$ can result in an increase or decrease of the jet core length, which has a significant influence on the jet breakup position, while its effects are vanishing in the 

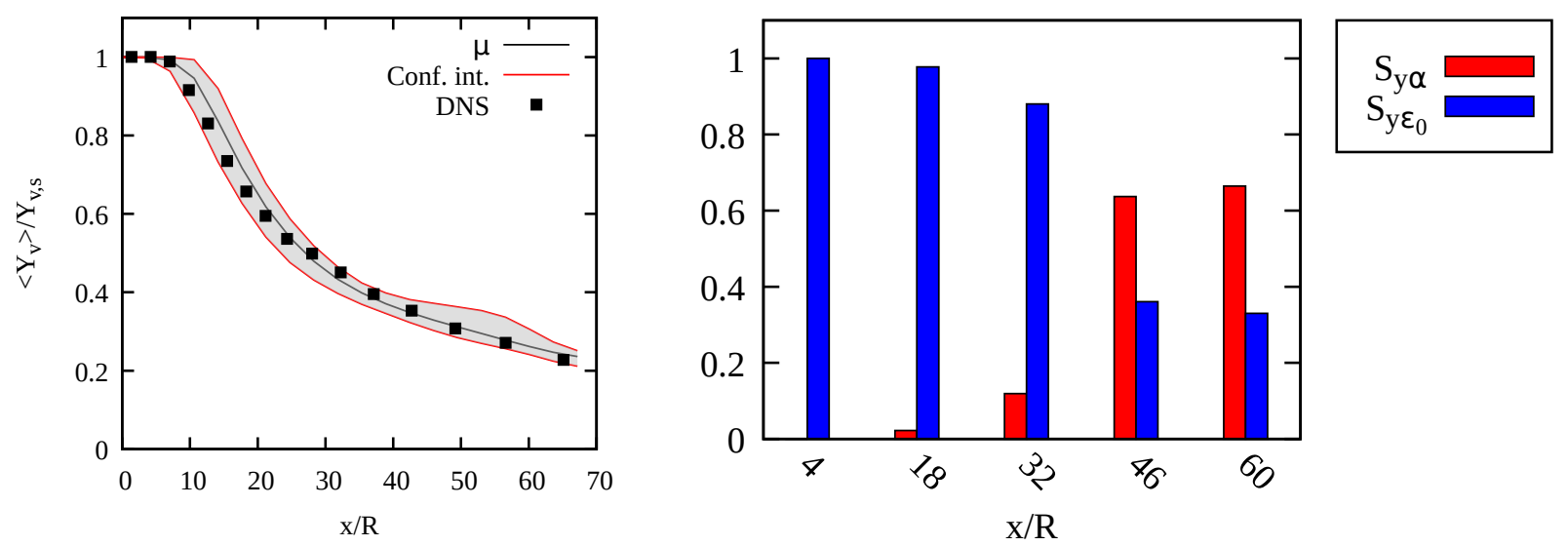

Fig. 2 Left: mean vapor concentration velocity. Red lines encompass a grey region, representing the confidence interval. The reference DNS data are reported as black dots. The vapor concentration is scaled by the saturated vapor mass fraction $Y_{v, s}$. Right: influence coefficients $S_{y \alpha}$ and $S_{y \epsilon_{0}}$ as a function of the nondimensional axial distance $x / R$.

far-field. Finally, very low or null values of $S_{u \alpha}$ are consistent with the DNS studies [14], highlighting that in the test case inquired, the droplets momentum is low and cannot significantly affect the axial velocity distribution along the jet axis.

The axial distributions of nondimensional vapor concentration $\left\langle Y_{v}\right\rangle / Y_{v, s}$ is shown in Fig. 2(left). Also in this case, the grey region encompassed by red lines represents the confidence interval, and the reference DNS data are reported as black dots. The agreement with the reference data is good. For what concerns the vapor mass fraction distribution, we observe that the confidence interval in the first ten radii from the inflow is very narrow, almost vanishing in the first five radii. This can be attributed to the saturated condition at the inflow, implying that the vapor mass fraction is at its maximum value and cannot increase, because evaporation cannot occur until dry air is mixed with the saturated vapor due to jet entrainment. A larger spread of the confidence interval is observed later on with the mean value being positioned in a symmetrical position with respect to the higher and lower bounds of the confidence interval until 25 radii. Further downstream, at 55 radii from the inflow, the upper limit of the confidence interval increases, highlighting that in the downstream region uncertainties in the models are amplified in terms of vapor mass fraction distribution. Based on the axial distribution of the influence coefficients in Fig. 2(right), we can observe how in the first part of the jet the $S_{y \alpha}$ is negligible, being the vapor mass fraction distribution influenced mostly by $\epsilon_{0}$, and hence by the velocity field. Thus, in the first 30 radii, the vapor mass fraction concentration spatial distribution is sensitive to the mean velocity field, while the dispersion model does not play any relevant role. This is because droplets in the proximity of the jet axis evaporate slowly, and the dispersion model is responsible for a change in their position, which is not large enough to imply a significant change in the experienced concentration evaporation. Moving away from the axis, $S_{y \alpha}$ increases, and $S_{y \epsilon_{0}}$ decreases. In this region, the velocity confidence interval is low, and the dispersion model can be responsible for an enhancement of droplets evaporation.

Similarly to what previously discussed for the axial distributions of mean axial velocity, the corresponding radial distributions are reported in the left column of Fig. 1. for nondimensional axial distances $x / R=20, x / R=40$, and $x / R=60$, from top to bottom, respectively. The agreement between the RANS confidence interval and the reference DNS data is satisfactory but not perfect. Moreover, the confidence interval spread diminishes moving towards the far-field, consistently with what observed in Fig. 1. and further shrinks moving away from the axis. The corresponding influence coefficients $S_{u \alpha}$ and $S_{u \epsilon_{0}}$ as a function of the radial distance are reported in the right column of Fig. 1 1 and show that, as expected, $S_{u \alpha}$ is null or negligible everywhere, confirming that the velocity distribution cannot be affected by the droplets momentum in the inquired conditions. This observation, along with the very narrow confidence intervals of Fig. 1 (left), leads to the conclusion that the two uncertain parameters $\alpha$ and $\epsilon_{0}$ have a negligible influence on the jet mixing layer behavior.

A different scenario is observed for the vapor mass fraction radial distributions reported in Fig. 4 (left) for nondimensional axial distances $x / R=20, x / R=40$, and $x / R=60$ from top to bottom, respectively. Also in this case, 

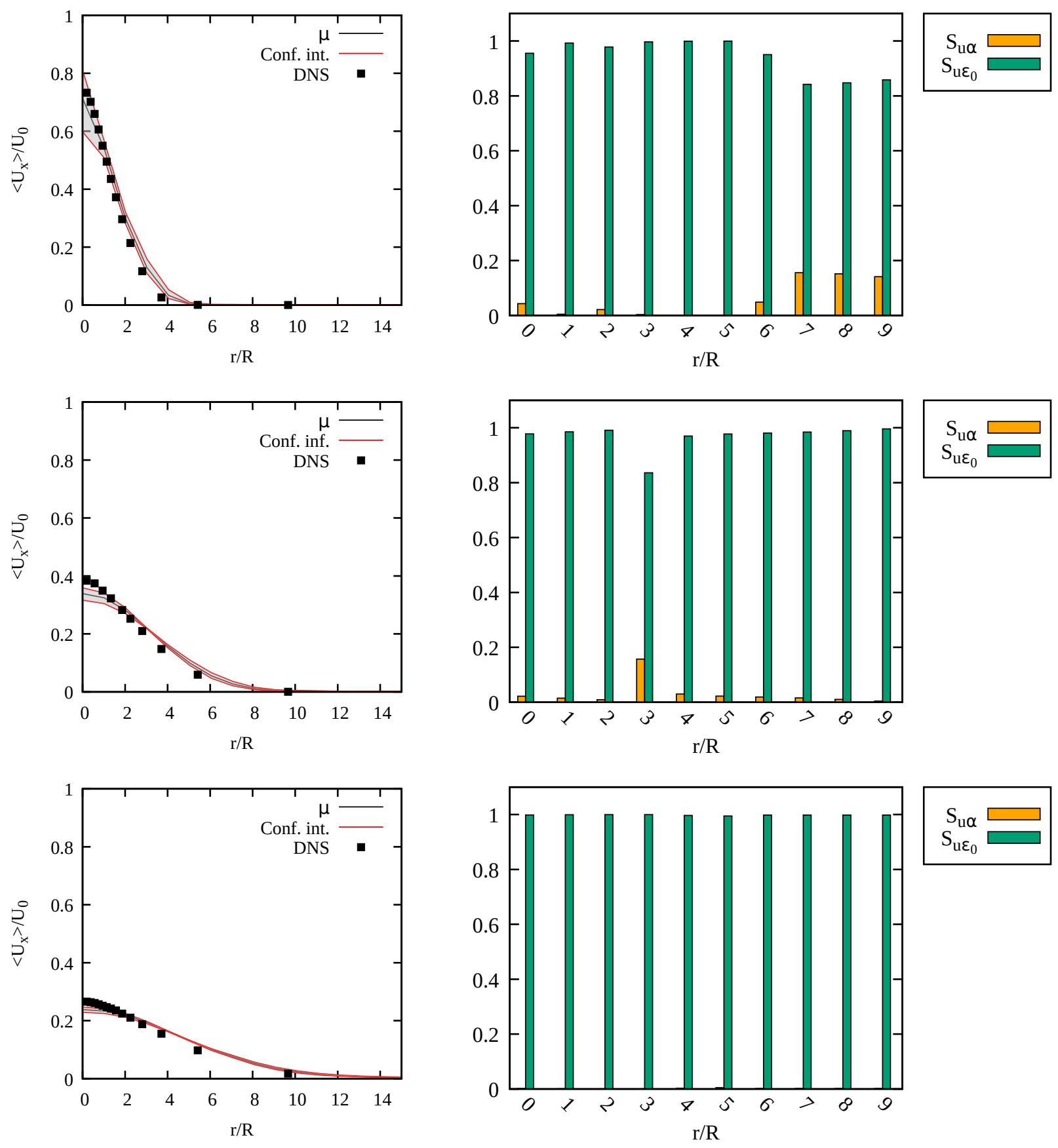

Fig. 3 Left column: mean axial velocity radial distribution. Red lines encompass a grey region, representing the confidence interval. The reference DNS data are reported as black dots. The axial velocity is scaled with the bulk inflow velocity $U_{0}$. Right column: influence coefficients $S_{u \alpha}$ and $S_{u \epsilon_{0}}$ as a function of the nondimensional axial distance $x / R$. From top to bottom: axial distances $x / R=20, x / R=40$, and $x / R=60$ 

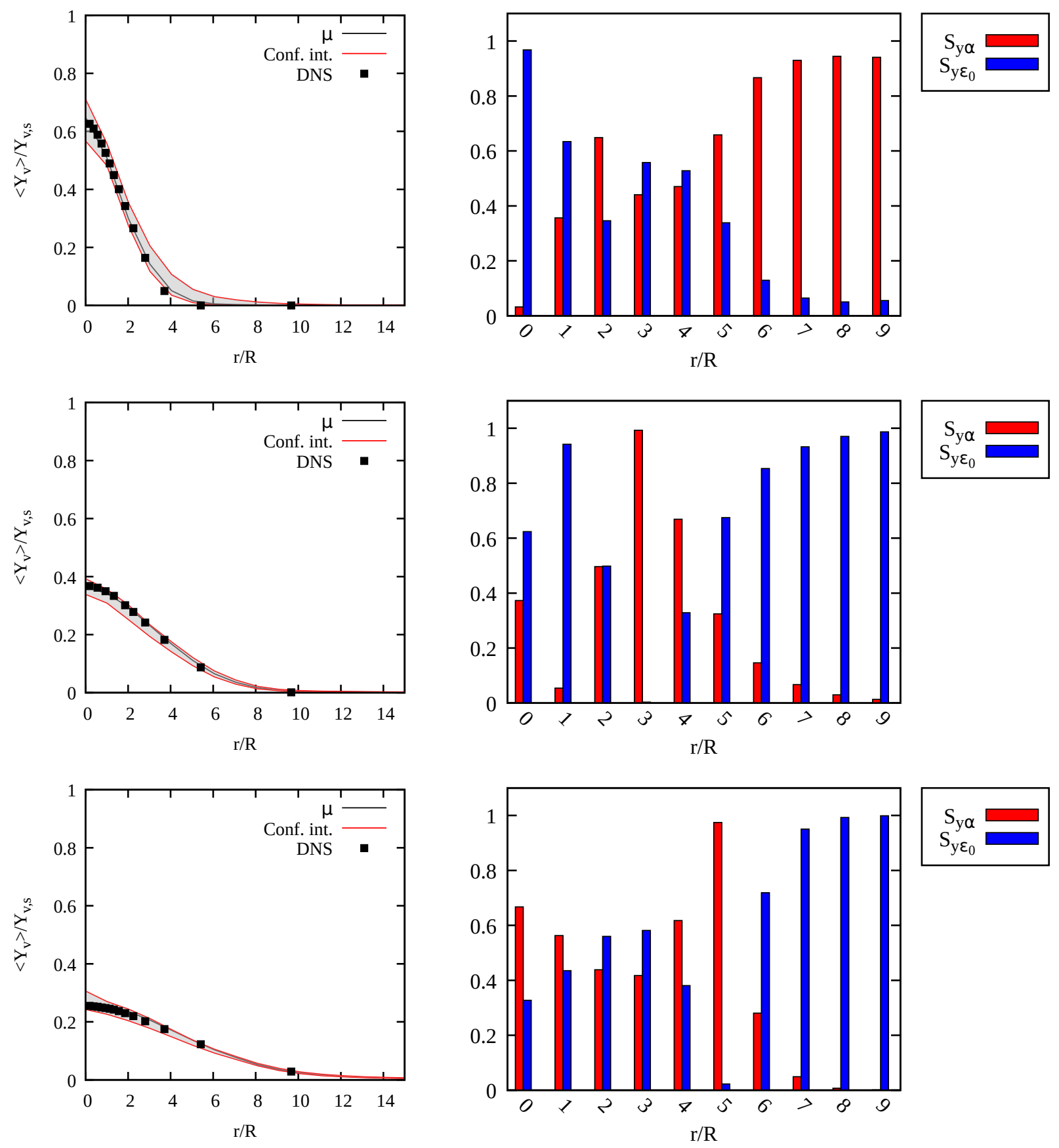

Fig. 4 Left column: mean vapor concentration radial distribution. Red lines encompass a grey region, representing the confidence interval. The reference DNS data are reported as black dots. The vapor concentration is scaled by the saturated vapor mass fraction $Y_{v, s}$. Right column: influence coefficients $S_{y \alpha}$ and $S_{y \epsilon_{0}}$ within the first nine radii from the centerline. From top to bottom: axial distances $x / R=20, x / R=40$, and $x / R=60$. 

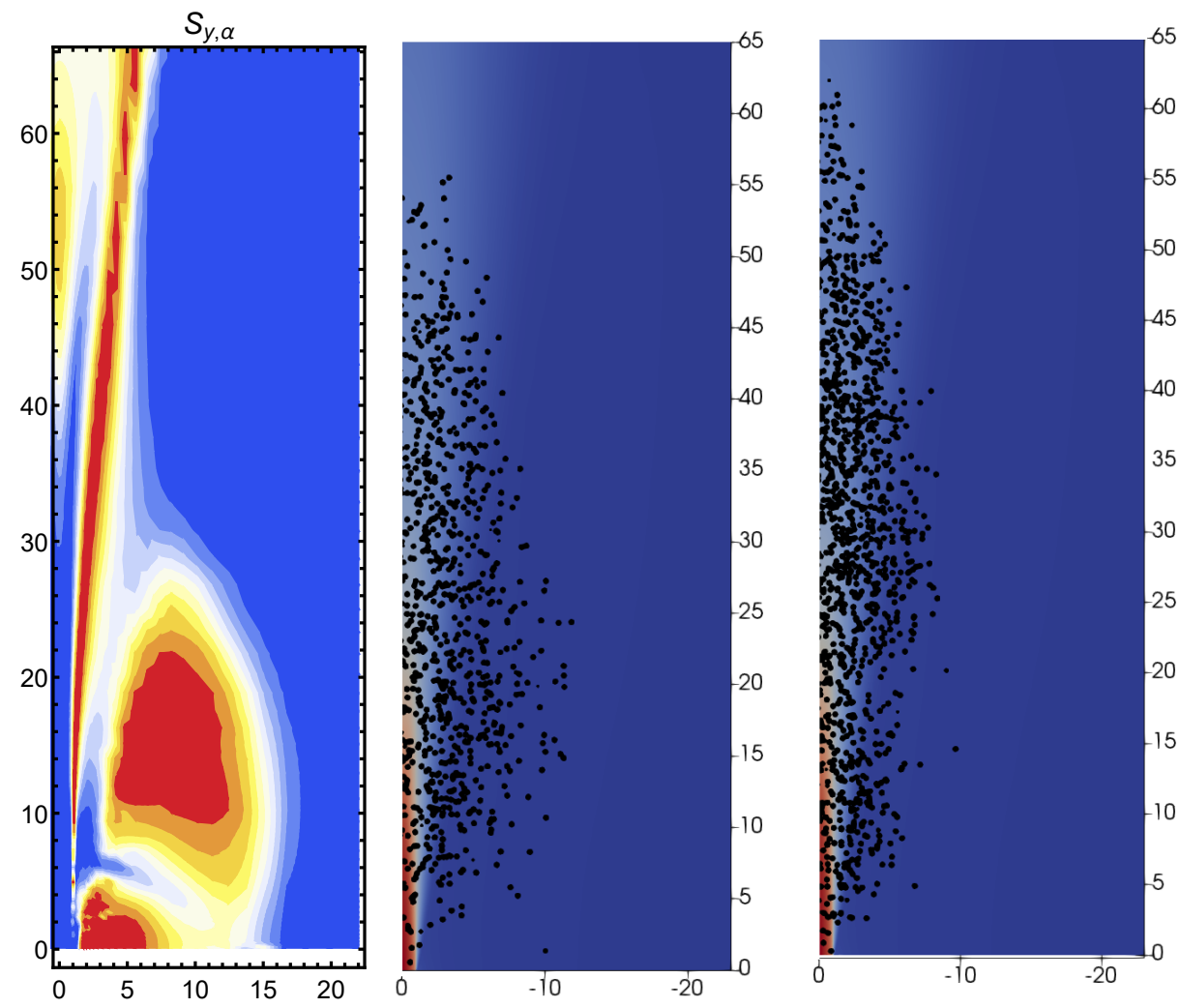

Fig. 5 Left: two dimensional distribution of the influence coefficient $S_{y \alpha}$ along a plane cutting the domain through the centerline. Center and right: particles instantaneous distribution for the stochastic dispersion model (center) and gradient-based dispersion model (right).

the agreement between the RANS confidence intervals and the reference DNS data is good, but vapor mass fraction shows broader confidence intervals in the entire radial range, especially for smaller distances from the inflow. The radial distributions of the influence coefficients $S_{y \alpha}$ and $S_{y \epsilon_{0}}$ are reported in the right column of Fig. 4 and show that for $x / R=20$ the vapor mass fraction depends only on $\epsilon$, that is, the dispersion model is not affecting the vapor mass fraction value in the core of the jet, as observed also for the axial distribution shown in Fig. 2. For axial distance $x / R=20$, moving away from the jet axis the role of $\epsilon_{0}$ decreases and the role of $\alpha$ becomes predominant. For larger axial distances, the role of $\alpha$ is maximum in the mixing layer $(r / R=3$ for $x / R=40$ and $r / R=5$ for $x / R=60)$ and then decreases.

This behavior is confirmed by the observation of the contour plot in Fig. 5 . The spatial distribution of $S_{y \alpha}$ is reported in the left, a snapshot of the vapor mass fraction and parcels distributions obtained employing the stochastic dispersion model is reported on the central panel of the same figure, while the solution obtained using the gradient-based dispersion model is reported on the right-side one. The dispersion model influences the vapor mass fraction in the shear layer. Here gradients of concentration are such that small perturbation in droplet position, due to the dispersion term, can imply a large variation in the temperature and vapor concentration experienced by the droplet, with a possibly strong impact on the evaporation. In Fig. 5(left), we can also notice a region, for $x / R<25$, where $S_{y \alpha}$ is high outside the mixing layer. Observing the instantaneous positions of the droplets in the two snapshots in the center- and right-side panels of Fig. 5. we can observe that the two dispersion models lead to different droplets spatial distributions in that region. Although in that region the mean vapor mass fraction is very low, and its confidence interval very narrow, the influence coefficients highlighted the difference in the droplet spatial distributions obtained by means of the two dispersion models.

The joint pdf $p\left(\left\langle U_{x}\right\rangle / U_{0},\left\langle Y_{v}\right\rangle / Y_{v, s}\right)$ is reported in Fig. 6(center) along with the marginal probabilities $p\left(\left\langle U_{x}\right\rangle / U_{0}\right)$ and $p\left(\left\langle Y_{v}\right\rangle / Y_{v, s}\right)$ in Fig. 6(left) and (right), respectively. Three axial distances are inquired $x / R=20,30$, and 60. The marginal pdf $p\left(\left\langle U_{x}\right\rangle / U_{0}\right)$ is shown to be consistent with the velocity behavior observed in Fig. 11, with the pdf getting narrower moving way from the jet inflow and remaining mostly symmetrical throughout the entire axial distance. Also the marginal pdf $p\left(\left\langle Y_{v}\right\rangle / Y_{v, s}\right)$ is consistent with the axial distribution shown in Fig. 2, with the pdf losing symmetry 

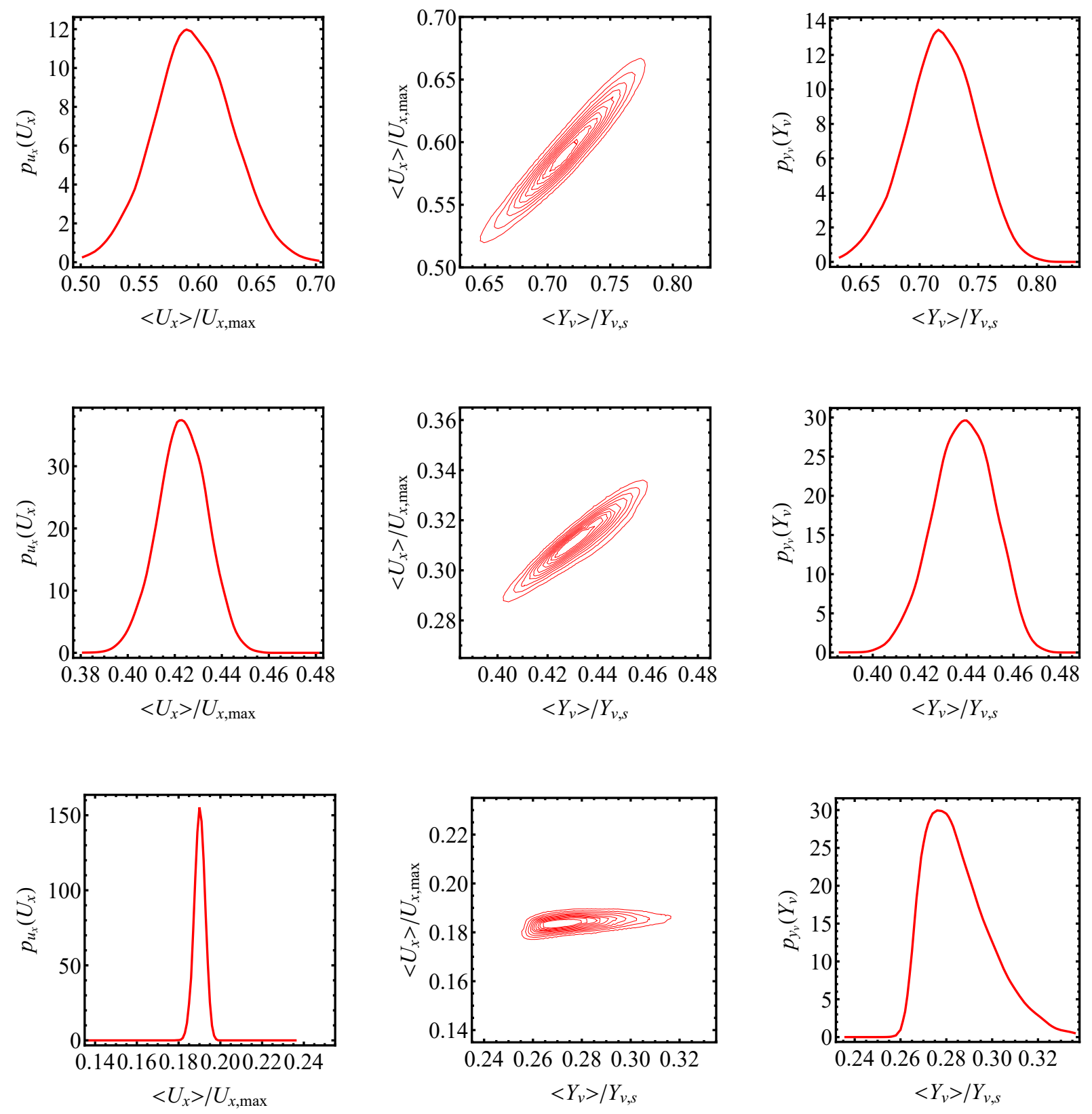

Fig. 6 Joint (center) and marginal (left, right) probability density functions of the mean axial velocity and vapor concentration re-scaled with their maximum values, namely the inflow velocity at the centerline $U_{x, \max }$ and the concentration of saturated vapor $Y_{v, s}$, at three different axial distances from the inflow: from top to bottom $x / R=20,30,60$. 
moving way from the jet inflow, and the most probable and the mean values getting closer to the lower bound of the confidence interval. Finally, the joint pdf allows us to point out an additional piece of information. At axial position $x / R=20$ the nondimensional velocity $\left\langle U_{x}\right\rangle / U_{0}$ and the scaled vapor mass fraction $\left\langle Y_{v}\right\rangle / Y_{v, s}$ are positively correlated. This means that an increase in the jet mean axial velocity leads to an increase in the vapor concentration, indicating that vapor concentration is strictly related to the mean velocity field. For larger distances from the jet inflow, the correlation decreases and is almost null for $x / R=60$. At this axial distance, the mean velocity pdf is narrow, and its correlation to vapor mass fraction is negligible, leading to the conclusion that the far-field velocity distribution is affected by neither the dispersion model nor the inflow value of dissipation $\epsilon_{0}$.

\section{Conclusions}

The work's scope was to assess the capabilities of uncertainty quantification techniques when dealing with low-fidelity models rich in uncertain parameters. Hence, a polynomial chaos expansion (PCE) technique was employed to generate surrogate models of the uncertain QoIs following a non-intrusive approach on RANS predictions of an acetone spray turbulent evaporating jet. The surrogate models were then employed to perform a sensitivity analysis of the velocity and vapor mass fraction distributions to the two uncertain parameters: the inflow value of the dissipation rate $\epsilon_{0}$ and a factor $\alpha$, which varies between zero and one and blends a stochastic and a gradient-based dispersion model. Although this is a preliminary feasibility study, far from the real application context, in which a greater variety of physical phenomena are involved (e.g., primary and secondary breakup and combustion), it proved to be very effective in pointing out the main features of the employed models. The main findings of this work are: (i) the velocity field is influenced by the uncertainty in $\epsilon_{0}$ mostly in the jet-core region, close to the inflow; (ii) the droplets momentum is unable to affect the velocity field considerably for the conditions that were studied; (iii) the vapor mass fraction is influenced by both the velocity field and the dispersion model; (iv) in the shear layers the dispersion model has a strong influence on the vapor mass concentration. Moreover, the addition of a confidence interval to the RANS results allows discriminating between those regions where modification in the parameters can better match the reference data and those regions where modification in the parameters of choice is not sufficient to attain a better agreement. Finally, the amount of information gained at a relatively low computational cost confirms the effectiveness of the UQ methods when dealing with design-oriented CFD approaches.

\section{Acknowledgments}

The authors acknowledge the support of the Italian Ministry of University and Research (MIUR) and King Abdullah University of Science and Technology OSR-2019-CCF-1975-35 Subaward Agreement. Computational resources were provided by the KAUST Supercomputing Laboratory (KSL).

\section{References}

[1] Ciottoli, P., Malpica Galassi, R., Lapenna, P., Leccese, G., Bianchi, D., Nasuti, F., Creta, F., and Valorani, M., "Systematic strategies for thermochemical model reduction in rocket propulsion applications," 2018 Joint Propulsion Conference, 2018.

[2] Boyette, W., Guiberti, T., Magnotti, G., and Roberts, W., "Structure of turbulent nonpremixed syngas flames at high pressure," Proceedings of the Combustion Institute, 2019.

[3] Lapenna, P., Lamioni, R., Ciottoli, P., and Creta, F., "Low-mach number simulations of transcritical flows," 2018.

[4] Lapenna, P., Ciottoli, P., and Creta, F., "Unsteady Non-Premixed Methane/Oxygen Flame Structures at Supercritical Pressures," Combustion Science and Technology, 2017.

[5] Li, Z., Malpica Galassi, R., Ciottoli, P., Parente, A., and Valorani, M., "Characterization of jet-in-hot-coflow flames using tangential stretching rate," Combustion and Flame, 2019.

[6] Ciottoli, P., Malpica Galassi, R., Angelilli, L., Cuoci, A., Im, H., and Valorani, M., "Analysis of Wall-flame Interaction in Laminar Non-premixed Combustion," Combustion Science and Technology, 2019.

[7] Martelli, E., Ciottoli, P., Bernardini, M., Nasuti, F., and Valorani, M., "Detached-eddy simulation of shock unsteadiness in an overexpanded planar nozzle," AIAA Journal, 2017.

[8] Martelli, E., Saccoccio, L., Ciottoli, P., Tinney, C., Baars, W., and Bernardini, M., "Flow dynamics and wall-pressure signatures in a high-Reynolds-number overexpanded nozzle with free shock separation," Journal of Fluid Mechanics, 2020. 
[9] Gargiulo, G., Ciottoli, P., Martelli, E., Malpica Galassi, R., and Valorani, M., "Numerical analysis of laser-pulse transient ignition of oxygen/methane mixtures in rocket-like combustion chamber," Acta Astronautica, 2019.

[10] Le Maître, O., and Knio, O., Multiresolution analysis for uncertainty quantification, 2017.

[11] Huan, X., Safta, C., Geraci, G., Eldred, M. S., Vane, Z. P., Lacaze, G., Oefelein, J. C., and Najm, H. N., "Global sensitivity analysis and estimation of model error, toward uncertainty quantification in scramjet computations," AIAA Journal, Vol. 56, No. 3, 2018, pp. 1170-1184. https://doi.org/10.2514/1.J056278.

[12] Malpica Galassi, R., Valorani, M., Najm, H., Safta, C., Khalil, M., and Ciottoli, P., "Chemical model reduction under uncertainty," Combustion and Flame, 2017.

[13] Dalla Barba, F., and Picano, F., "Clustering and entrainment effects on the evaporation of dilute droplets in a turbulent jet," Physical Review Fluids, Vol. 3, No. 3, 2018. https://doi.org/10.1103/PhysRevFluids.3.034304.

[14] Ciottoli, P., Battista, F., Malpica Galassi, R., Dalla Barba, F., and Picano, F., "Direct numerical simulations of the evaporation of dilute sprays in turbulent swirling jets," Flow Turbulence and Combustion, 2020.

[15] Jenny, P., Roekaerts, D., and Beishuizen, N., "Modeling of turbulent dilute spray combustion," Progress in Energy and Combustion Science, Vol. 38, 2012, pp. 846-887. https://doi.org/10.1016/j.pecs.2012.07.001

[16] Elghobashi, S., and Truesdell, G. C., "On the two-way interaction between homogeneous turbulence and dispersed solid particles. I: Turbulence modification,” Physics of Fluids A: Fluid Dynamics, Vol. 5, No. 7, 1993, pp. 1790-1801. https://doi.org/10.1063/1.858854

[17] Elghobashi, S., Particle-laden turbulent flows: direct simulation and closure models, chapter and pages, pp. 91-104. https://doi.org/10.1007/978-94-011-3632-7_4

[18] Balachandar, S., and Eaton, J. K., "Turbulent Dispersed Multiphase Flow," Annual Review of Fluid Mechanics, Vol. 42, No. 1, 2010, pp. 111-133. https://doi.org/10.1146/annurev.fluid.010908.165243

[19] Chen, X.-Q., and Pereira, J. C., "Prediction of evaporating spray in anisotropically turbulent gas flow," Numerical Heat Transfer, Part A: Applications, Vol. 27, No. 2, 1995, pp. 143-162. https://doi.org/10.1080/10407789508913693

[20] Chen, X.-Q., and Pereira, J., "Computation of turbulent evaporating sprays with well-specified measurements: a sensitivity study on droplet properties," International Journal of Heat and Mass Transfer, Vol. 39, No. 3, 1996, pp. 441 - 454. https://doi.org/https://doi.org/10.1016/0017-9310(95)00162-3.

[21] Pope, S., "An explanation of the turbulent round-jet/plane-jet anomaly," AIAA Journal, 1978.

[22] Shih, T.-H., Liou, W. W., Shabbir, A., Yang, Z., and Zhu, J., "A new k- eddy viscosity model for high reynolds number turbulent flows," Computers Fluids, Vol. 24, No. 3, 1995, pp. 227 - 238. https://doi.org/https://doi.org/10.1016/0045-7930(94)00032-T

[23] Gosman, A. D., and loannides, E., “Aspects of Computer Simulation of Liquid-Fueled Combustors," Journal of Energy, Vol. 7 , No. 6, 1983, pp. 482-490. https://doi.org/10.2514/3.62687

[24] Mueller, M., and Raman, V., "Model form uncertainty quantification in turbulent combustion simulations: Peer models," Combustion and Flame, Vol. 187, 2018, pp. 137-146. https://doi.org/10.1016/j.combustflame.2017.09.011 